\title{
A CRIAÇÃO DO CONHECIMENTO ORGANIZACIONAL SOB A PERSPECTIVA DAS TEORIAS DESENVOLVIMENTISTAS
}

\section{ORGANIZATIONAL KNOWLEDGE CREATION IN THE FRAMEWORK OF DEVELOPMENTAL THEORIES}

\author{
Carlos Henrique Prim ${ }^{1}$; Cristiano José Castro de Almeida Cunha ${ }^{2}$ \\ ${ }^{1}$ Universidadade Federal de Santa Catarina - UFSC - Florianópolis - Brasil chprim@egc.ufsc.br \\ ${ }^{2}$ Universidadade Federal de Santa Catarina - UFSC - Florianópolis - Brasil cunha@deps.ufsc.br
}

\begin{abstract}
Resumo
O padrão de interação entre as organizações da era digital é caracterizado pela não-linearidade. Isso gera um ambiente que exige das organizações a contínua criação de conhecimento. Esta exigência demanda mudanças no comportamento e atuação dos indivíduos, tendo repercussões na criação do conhecimento organizacional. Partindo dessa constatação, o presente trabalho sugere a aplicação das teorias do desenvolvimento do indivíduo na criação do conhecimento organizacional. Por meio dessa aplicação, as organizações podem tanto otimizar a criação do conhecimento quanto levar em consideração o desenvolvimento do indivíduo. As principais teorias do desenvolvimento são introduzidas. A sua aplicabilidade é demonstrada no desenvolvimento da liderança. Com o embasamento teórico, faz-se uma análise dos quatro modos de conversão do conhecimento sob a perspectiva do modelo desenvolvimentista. Uma das conclusões do trabalho é que, quando se considera o indivíduo, a capacidade de criação de conhecimento explícito é limitada por uma contrapartida tácita. Constata-se também que a perspectiva desenvolvimentista permite um melhor ajuste entre o desenvolvimento das competencias individuais e os objetivos organizacionais.
\end{abstract}

Palavras-chave: criação de conhecimento, teorias do desenvolvimento, liderança, sistemas nãolineares.

\section{Introdução}

As organizações vivem um dilema. Por um lado, devem se adaptar às mudanças ambientais pela contínua criação do conhecimento organizacional. Por outro, dependem da produtividade de indivíduos que sentem os impactos de tais adaptações, com conseqüências negativas sobre a criação do conhecimento. O objetivo do presente trabalho é apresentar uma análise deste dilema com base nas teorias do desenvolvimento do indivíduo, e ao mesmo tempo, apresentar uma nova perspectiva teórica que possibilite a otimização da criação do conhecimento organizacional e proporcione um melhor ajuste dos indivíduos às organizações. 
O trabalho tem caráter exploratório e leva em consideração diferentes áreas do conhecimento na elaboração de suas hipóteses. As áreas abordadas ao longo do trabalho são: sistemas complexos, teorias do desenvolvimento do indivíduo, desenvolvimento da liderança e criação do conhecimento organizacional. Para se chegar ao objetivo proposto, foram utilizadas obras de referência relacionadas às diferentes áreas incluídas no estudo, além de artigos encontrados em pesquisa bibliográfica pelos principais periódicos científicos.

A primeira seção do trabalho justifica a afirmativa de que a atual dinâmica das organizações tem reflexos na vida dos indivíduos que a compõem. Demonstra-se que isso é conseqüência das interações não-lineares entre as organizações da era digital, cujos padrões de comportamento têm impactos no nível individual. Em função desses impactos, são necessárias teorias que considerem o indivíduo a partir de uma perspectiva dinâmica. Estas teorias são apresentadas na seção três.

As duas seções seguintes tratam das aplicações dessas teorias nas organizações. A primeira delas apresenta uma abordagem em uso para o desenvolvimento da liderança, que toma como base algumas das principais suposições das teorias do desenvolvimento do indivíduo. A segunda investiga a criação do conhecimento organizacional sob a perspectiva das teorias desenvolvimentistas. Os quatro modos de conversão do conhecimento de Nonaka e Takeuchi (1997) são analisados nessa perspectiva. Por fim, algumas conclusões são delineadas.

\section{As demandas da nova sociedade}

A conectividade digital, promovida pela disseminação das tecnologias de informação e comunicação, reduziu drasticamente as barreiras existentes no mercado (EVANS; WURSTER, 1997). Tal acontecimento modificou a dinâmica do sistema, que se tornou não-linear. Uma interação entre dois agentes é não-linear quando as ações de um têm conseqüências no outro, que então reage afetando o primeiro. Este, em seguida, adota uma nova ação, e assim por diante (STACEY, 1995). Sistemas cujos padrões de interação são não-lineares tornam-se imprevisíveis.

Uma das características de sistemas não-lineares, de acordo com as ciências da complexidade, é sua natureza holográfica ou fractal (STACEY, 1996). Um fractal é um padrão reconhecível que se repete em diferentes níveis. Nesse sentido, uma organização é um sistema que é parte de um sistema maior, a sociedade. Por outro lado, é formada por partes que também são sistemas. Considerando a existência dos diferentes níveis, diz-se que as partes continuamente recriam o todo, e o todo afeta o comportamento das partes (STACEY, 1996).

Os indivíduos, por exemplo, podem ser modelados por símbolos, cujo padrão assume a

forma de regras, modelos mentais, roteiros de condutas e paradigmas. Esse padrão forma um esquema que determina o seu comportamento (STACEY, 1996). No que se refere às organizações, 
seus esquemas são construídos a partir dos padrões dos pensamentos e comportamentos dos indivíduos. Ou seja, modificações nos esquemas individuais recriam os esquemas organizacionais. Sendo a organização parte de um sistema não-linear, deve continuamente rever e modificar os seus esquemas para garantir sua sobrevivência. Contudo, tais modificações acontecem pela alteração dos esquemas dos indivíduos que a constituem. Portanto, modificações no âmbito organizacional afetam o comportamento individual, fechando assim um ciclo de interferência mútua entre indivíduo e organização.

As inovações tecnológicas reforçaram este ciclo, gerando uma demanda contínua por modificações dos esquemas dos indivíduos. Para lidar com esta nova configuração, tornam-se necessárias teorias que sejam capazes de explicar a dinâmica do desenvolvimento do indivíduo e que, ao mesmo tempo, possam auxiliar as organizações a estruturarem seus processos de geração de conhecimento. A próxima seção introduz algumas das principais teorias do desenvolvimento humano que podem ser aplicadas com esse propósito.

\section{A evolução das teorias do desenvolvimento}

Piaget foi um dos pioneiros na elaboração de uma teoria do desenvolvimento em estágios. $\mathrm{O}$ foco de seus estudos foi o desenvolvimento físico-cognitivo de crianças. Outros pesquisadores, muitos dos quais influenciados pelos trabalhos de Piaget, desenvolveram abordagens alternativas para explicar o fenômeno. As teorias diferem entre si em aspectos tais como a interpretação do fenômeno, a caracterização dos estágios e o grau de embasamento empírico-teórico. $\mathrm{O}$ entendimento dessas diferenças é fundamental para que as teorias possam ser avaliadas e eficientemente aplicadas na prática. O objetivo ao se apresentar, a seguir, algumas das principais teorias do desenvolvimento, é evidenciar essas diferenças. Algumas conclusões quanto à aplicação das teorias de desenvolvimento são apresentadas ao final da seção.

Erikson (1998) desenvolveu uma teoria que leva em consideração três diferentes dimensões do desenvolvimento: a física, a cognitiva e a social. Qualquer mudança em uma dessas dimensões tem repercussões nas outras. A ponte entre elas é a construção de significados. Por exemplo, uma pessoa, ao interagir com outra (dimensão social), constrói significados dessa experiência. Isso, por sua vez, influenciará, de alguma forma, tanto o seu desenvolvimento físico quanto o cognitivo. Originalmente, a teoria apresentava oito fases de desenvolvimento distribuídas por faixas etárias ao longo de toda a vida. Uma nona fase foi posteriormente acrescentada. A definição e a descrição dos estágios foram realizadas exclusivamente a partir das observações pessoais de Erikson. Ou seja, a teoria não possui embasamento empírico, dificultando sua validação. 
Loevinger (1993), por sua vez, elaborou sua teoria com base em resultados empíricos. Ela usou uma metodologia para a identificação do estágio de desenvolvimento do respondente. Essa ferramenta - o Washington University Sentence Completion Test (WUSCT) - é constituída por sentenças a serem completadas pelo respondente. Nela, parte-se do princípio que as respostas revelam a forma como o respondente constrói os significados de suas experiências, e que esta forma caracteriza o seu estágio de desenvolvimento. O método consiste em classificar as repostas em categorias associadas aos diferentes estágios. O desenvolvimento do WUSCT possibilitou que os estágios fossem bem caracterizados. Por outro lado, a caracterização mais apurada dos estágios permitiu que a própria ferramenta fosse aperfeiçoada. Portanto, os desenvolvimentos da teoria de Loevinger e do WUSCT aconteceram em paralelo, um em função do outro (ISHIKAWA, 2005).

Apesar de ter contribuído para a taxonomia dos estágios, a teoria de Loevinger deixou a desejar quanto à interpretação do fenômeno (ISHIKAWA, 2005). Um importante avanço nesse sentido foi proporcionado por Kegan (1982). De acordo com esse pesquisador, o que rege o processo de desenvolvimento é a capacidade de construir significados, que abrange todas as demais dimensões da experiência humana tais como a cognitiva, a emocional e a social. Essa capacidade é desenvolvida ao longo da vida, inclusive na fase adulta, através de uma seqüência de transferências, que caracterizam as transições de estágio, quando o que era sujeito passa a ser objeto. Por exemplo, uma criança na fase Imperial constrói significados através de suas necessidades (sujeito). Na fase seguinte, Interpessoal, ao invés de construir significados através de suas necessidades, ela passa a tê-las (objeto). Nessa nova fase, ela constrói significados através dos seus relacionamentos - uma forma mais complexa de construir significados do que a anterior. A transição para um novo estágio somente ocorre após um período de amadurecimento no estágio anterior, dentro de um contexto adequado. O desenvolvimento também se caracteriza por uma alternância entre estágios de diferenciação e integração - o primeiro tendo um foco maior no indivíduo e o segundo nos relacionamentos.

Partindo da interpretação de Kegan e utilizando o WUSCT, Cook-Greuter (1999) fez um refinamento da teoria, à qual acrescentou novos estágios. As suposições de sua abordagem contém várias das proposições das teorias anteriormente comentadas. As principais delas são (COOKGREUTER, 2004):

- Estágios mais avançados são atingidos somente passando-se através dos estágios mais básicos. Uma vez que um estágio tenha sido "atravessado", ele passa a fazer parte do repertório de respostas de um indivíduo.

- Cada novo estágio inclui e transcende o estágio anterior. Isto é, as perspectivas anteriores permanecem partes da experiência e do conhecimento atual.

- Uma pessoa que tenha atingido um estágio mais avançado pode entender as visões de 
mundo dos estágios anteriores, mas uma pessoa que se encontra em um estágio mais básico não é capaz de entender as que se encontram nos estágios mais avançados.

- O desenvolvimento ocorre pela ação recíproca entre a pessoa e o ambiente. Ele pode ser encorajado e facilitado pelo desafio e por um suporte apropriado.

Cook-Greuter (2004) faz uma distinção entre desenvolvimento horizontal ou lateral e desenvolvimento vertical. O primeiro, mais comum, acontece com o apoio da educação formal, treinamento e outras formas usuais de aprendizagem. Esse desenvolvimento expande a capacidade de construir significados, mas o indivíduo permanece no mesmo estágio. Por outro lado, o desenvolvimento vertical, mais raro, caracteriza-se pela transição de um estágio para outro. $\mathrm{O}$ efeito desse desenvolvimento é bem mais significativo, pois uma nova capacidade de construir significados, mais ampla e complexa do que a anterior, é desenvolvida.

As quatro teorias apresentadas anteriormente evoluíram em diferentes direções. Um dos pontos positivos da teoria de Erikson, por exemplo, foi a inclusão da dimensão social como um aspecto fundamental do desenvolvimento. Loevinger fez importantes contribuições, tanto pelo embasamento empírico, quanto pelo desenvolvimento de uma ferramenta confiável de mensuração do estágio. Kegan, por sua vez, forneceu uma interpretação que explicita o mecanismo de transição entre um estágio e outro, bem como apresentou a lógica subjacente de cada estágio. Por fim, CookGreuter fez um refinamento da teoria, levando em consideração as principais características das demais abordagens. Com esses avanços, as teorias de desenvolvimento apresentam-se suficientemente embasadas a fim de que possam ser aplicadas nas organizações. Um exemplo de uma aplicação em uso é no desenvolvimento da liderança, conforme apresentado a seguir.

\section{Uma aplicação: o desenvolvimento da liderança}

Partindo do princípio que a capacidade de construir significados é o fator que mais limita a efetividade de um líder, Fisher et al. (2003) apresentam uma metodologia para o desenvolvimento da liderança - o questionamento na ação (action inquiry). O uso da metodologia permite que o líder engaje-se na investigação de suas intenções, estratégias e ações no momento da ação, utilizando como referencial os resultados de suas próprias ações. Isso provoca no líder uma ampliação de consciência quanto às suas premissas pessoais. Ao alinhar suas ações às estratégias e intenções reveladas durante o processo, o líder cria condições para o seu desenvolvimento vertical.

A metodologia apóia-se nas suposições das principais teorias do desenvolvimento, especialmente as de Cook-Greuter. Nela, os estágios de desenvolvimento são diretamente 
associados a diferentes categorias de liderança ${ }^{1}$. Segundo Cook-Greuter, há sete categorias comumente encontradas nas organizações: Oportunista, Diplomata, Especialista, Realizador, Individualista, Estrategista e Alquimista. O objetivo da metodologia é que o líder alcance o estágio Estrategista, pois é a partir desse ponto que ele adquire potencial para promover as necessárias transformações pessoais e organizacionais (ROOKE, 1997; ROOKE; TORBERT, 1998). A seguir, são descritas as sete categorias consideradas no desenvolvimento da liderança (FISHER; ROOKE; TORBERT, 2003; ROOKE; TORBERT, 1998):

- Oportunista: Interpreta a realidade pela perspectiva de suas próprias necessidades e desejos. Valoriza os resultados de curto prazo, apreciando, sobretudo, os aspectos financeiros e de poder da organização.

- Diplomata: Enxerga o mundo através dos seus relacionamentos. Adota os valores do grupo e segue fielmente suas normas. Contudo, evita qualquer tipo de conflito a fim de manter a harmonia no ambiente de trabalho.

- Especialista: Interpreta a realidade pela lógica da sua área de competência. Utiliza o raciocínio para atingir os seus objetivos. Valoriza o trabalho bem feito dos subordinados. Contudo, possui expectativas elevadas, podendo criar um ambiente de trabalho competitivo.

- Realizador: Interpreta o mundo através da perspectiva organizacional. Cria um clima de trabalho positivo, valorizando o trabalho em equipe. Presta atenção nas diferenças de pontos de vistas. No entanto, usualmente inibe o pensamento que esteja fora do convencional.

- Individualista: Reconhece que nenhuma forma de construir significados é natural. São, todas, interpretações do próprio indivíduo. Valoriza menos as regras externas e mais os princípios pessoais. É hábil em levar em consideração diferentes pontos de vista.

- Estrategista: Percebe o mundo em desenvolvimento e está engajado na sua transformação. Procura renovar sua forma de enxergar o mundo, bem como o das pessoas ao seu redor. Possui uma maior consciência de si e dos outros.

- Alquimista: Tem uma grande capacidade de renovar a si mesmo e às organizações. É capaz de lidar simultaneamente com várias situações em diversos níveis. É, em geral, carismático, ciente e guiado por altos padrões morais.

\footnotetext{
${ }^{1}$ Ao investigar a relação entre o desenvolvimento da liderança e a teoria do desenvolvimento, Ishikawa (2005) notou que os autores da abordagem do questionamento na ação relacionam diretamente as categorias de liderança com os estágios de desenvolvimento. No entanto, outros fatores da liderança tais como os motivacionais e os de conteúdo são desconsiderados. Ishikawa concluiu que a evolução entre os estágios é uma condição necessária, mas não suficiente, para o desenvolvimento da liderança.
} 


\section{Contribuição dos modelos de desenvolvimento para a criação de conhecimento}

As teorias de desenvolvimento podem ser aplicadas na criação do conhecimento organizacional. Tal aplicação pode auxiliar as empresas a serem mais eficientes nesse processo, bem como facilitar as mudanças a nível individual. A fim de explicitar as implicações dessa aplicação tanto para as organizações quanto para os indivíduos, os quatro modos de conversão do conhecimento de Nonaka e Takeuchi (1997) são analisados a seguir sob o ponto de vista das teorias desenvolvimentistas.

Socialização (tácito-tácito). Considerando que tanto aprendiz quanto mestre possuem uma certa capacidade de construir significados, três diferentes cenários podem ocorrer na socialização. No primeiro, ambos possuem a mesma capacidade de construir significados. Nesse caso, o aprendiz terá plenas condições de aprender pela observação e, com o tempo, ter um desempenho similar ao do mestre, pois ambos possuem estruturas equivalentes de construção de significados. $\mathrm{O}$ segundo cenário é aquele no qual o aprendiz está em um estágio de desenvolvimento inferior ao do mestre. Nesta situação, o aprendiz poderá ter dificuldades para interpretar as ações observadas, tornando o seu aprendizado mais lento. Uma vez habilitado, poderá não ter um desempenho equivalente ao do mestre, pois sua capacidade de interpretar a realidade é mais limitada. No terceiro cenário, o aprendiz possui uma capacidade de construir significados superior ao do mestre. Desta vez, ele poderá apresentar uma maior facilidade para interpretar as ações do mestre e, portanto, deverá aprender mais rápido. $\mathrm{O}$ aprendiz poderá, ainda, acrescentar novos significados ao conhecimento.

Externalização (tácito-explícito). O desenvolvimento da capacidade de construir significados está estreitamente relacionada com a linguagem. É neste princípio que está fundamentada a ferramenta de mensuração de estágio - o WUSCT. Ao mudar de estágio, uma pessoa tem condições de perceber e descrever uma nova realidade, mais ampla, que antes não era percebida. Ou seja, sua capacidade de conceitualizar o conhecimento tácito em explícito, através de metáforas, analogias ou alguma outra forma, amplia-se com o desenvolvimento entre os estágios.

A externalização também depende da interação entre indivíduos, pois os conceitos são usualmente formados pelo diálogo ou reflexão coletiva. A capacidade de interagir amplia-se à medida em que alguém se desenvolve entre os diferentes estágios. Esta afirmativa pode ser validada por meio da análise dos padrões de feedback das diferentes categorias de desenvolvimento da liderança, apresentados na tabela 1. Verifica-se que a abertura para dar e receber feedback aumenta ao longo dos estágios, fazendo com que o padrão de diálogo seja cada vez mais não-linear, portanto mais complexo e criativo, ampliando a capacidade do líder de interagir com indivíduos em diferentes estágios. 
Tabela 1 - Estágios de desenvolvimento e padrão de feedback.

\begin{tabular}{|c|l|}
\hline \multicolumn{1}{|c|}{ Estágio } & \multicolumn{1}{c|}{ Feedback } \\
\hline Oportunista & Reage ao feedback como um ataque ou ameaça. \\
\hline Diplomata & Recebe feedback com desaprovação, ou como algo que lembra as normas. \\
\hline Especialista & $\begin{array}{l}\text { Leva o feedback para o lado pessoal, defendendo sua posição. Coloca de lado o } \\
\text { feedback daqueles que não são vistos como especialistas da sua área. }\end{array}$ \\
\hline Realizadora & $\begin{array}{l}\text { Aceita o feedback, especialmente se lhe ajudar a atingir seus objetivos e o seu } \\
\text { aperfeiçoamento. }\end{array}$ \\
\hline Individualista & $\begin{array}{l}\text { Acolhe o feedback como uma necessidade para o seu auto-conhecimento e para } \\
\text { descobrir aspectos escondidos do seu comportamento. }\end{array}$ \\
\hline Estrategista & $\begin{array}{l}\text { Convida o feedback para a auto-atualização, pois o conflito é visto como um } \\
\text { aspecto inevitável das relações. }\end{array}$ \\
\hline Alquimista & $\begin{array}{l}\text { Vê o feedback como parte natural do sistemas vivos, essencial para a } \\
\text { aprendizagem e mudança. }\end{array}$ \\
\hline
\end{tabular}

Fonte: COOK-GREUTER, 2004

Combinação (explícito-explícito). Pelos mesmos motivos apresentados na externalização, quanto mais uma pessoa avança nos estágios, maior a sua capacidade de associar diferentes tipos de conhecimento para a criação de um novo conhecimento. Analisando-se as categorias de desenvolvimento da liderança, conclui-se que o estágio Individualista é um importante ponto de inflexão, pois é a partir desse estágio que o líder é capaz de lidar com pontos de vista distintos para criar uma visão compartilhada. Isso indica que, a partir desse estágio, a combinação começa a ser realizada com mais eficiência.

Internalização (explícito-tácito). Pessoas que possuem diferentes capacidades de construir significados desenvolvem e internalizam atributos diferenciados ao realizarem uma mesma atividade. Sandberg (2000) fornece um exemplo de como isso acontece na prática. Ele investigou a competência humana no trabalho utilizando uma abordagem interpretativa. Foram coletadas descrições da experiência vivida de vinte engenheiros de uma indústria automobilística, cujo trabalho era otimizar diferentes características de um motor, tais como o consumo, a emissão e a potência. A otimização era realizada pelo ajuste de diferentes parâmetros num sistema de monitoramento eletrônico.

Ao analisar as descrições, o pesquisador identificou três formas qualitativamente diferentes de realizar o trabalho. Na primeira delas, os engenheiros otimizavam uma única característica do motor de cada vez até que todas estivessem de acordo com as normas requeridas. Uma segunda forma de conceber o trabalho era realizada por engenheiros que levavam em consideração todas as etapas da otimização ao mesmo tempo. Esses engenheiros possuíam uma visão sistêmica do motor, percebendo a interdependência de suas diferentes características. Por fím, a terceira forma identificada dizia respeito ao trabalho de engenheiros que otimizavam o motor sob a perspectiva do cliente. Ou seja, os engenheiros dessa concepção relacionavam a sua atividade com a satisfação do cliente. 
O autor distinguiu atributos específicos para cada uma das três concepções identificadas. Foi verificado que os engenheiros da segunda concepção podiam, em situações específicas, utilizar os atributos da primeira, mas não o contrário. Semelhantemente, os engenheiros da terceira concepção eram capazes de agir de acordo com qualquer uma das duas primeiras concepções. Novamente, o contrário não ocorreu. $\mathrm{O}$ autor também notou que as diferenças de competência não estavam diretamente relacionadas com a educação formal nem com o tempo de profissão, e que um engenheiro poderia progredir da condição de novato para a de expert sem mudar a sua concepção de trabalho. Por fim, sugeriu duas formas de desenvolvimento da competência: a primeira, pela mudança de concepção (desenvolvimento vertical) e a segunda, pelo aperfeiçoamento da concepção atual (desenvolvimento horizontal).

Apesar de Sandberg não ter relacionado o seu estudo com as teorias do desenvolvimento do indivíduo, fica evidente, pelo que foi exposto, que os engenheiros das três categorias identificadas estão em estágios de desenvolvimento diferentes. Os engenheiros da terceira concepção estão num estágio mais avançado e internalizaram os atributos relacionados aos dois estágios anteriores. Conclui-se, então, que os engenheiros de uma concepção podem internalizar apenas os atributos relacionados ao estágio em que se encontram. Ou, de forma alternativa, a mudança de estágio proporciona a internalização dos atributos relacionados à concepção atual.

\section{Conclusões}

Neste artigo, procuramos mostrar que a abordagem desenvolvimentista pode contribuir para a criação do conhecimento organizacional e para o aperfeiçoamento das teorias subjacentes.

Um dos resultados da pesquisa mostra que o conhecimento explícito, de forma geral, parece que pode ser trabalhado de forma mais eficiente por indivíduos em estágios mais avançados, quando a capacidade cognitiva e de interação são mais desenvolvidas. Por outro lado, a mudança de estágio, necessária para o avanço dessas capacidades, caracteriza um processo de internalização, quando os atributos atuais passam a fazer parte do repertório de respostas do indivíduo. Portanto, a continuidade do desenvolvimento da capacidade de explicitar o conhecimento depende de contrapartidas tácitas, que ocorrem pela internalização de conhecimento nas mudanças de estágio.

Além disto, a teoria do desenvolvimento permite sugerir que as equipes de criação de conhecimento devem ter melhor desempenho quando formadas por pessoas em diferentes estágios. Pessoas nos estágios básicos são mais envolvidas nos detalhes de uma dada situação ou área de conhecimento. Dessa forma, podem fornecer idéias para que outros, com um maior conhecimento contextual (estágios mais avançados), apliquem-nas apropriadamente. Essas equipes devem ser 
lideradas por alguém capaz de criar um clima de confiança mútua entre os seus integrantes.

As competências da organização, por sua vez, podem ser mais bem geridas considerando-se as diferentes capacidades de construir significados. Existe uma relação entre a qualidade da realização de uma tarefa e o estágio de desenvolvimento do indivíduo - determinadas tarefas são melhor realizadas por indivíduos que estão no estágio $\mathrm{X}$ do que por indivíduos que estão no estágio Y. No estudo de Sandberg, por exemplo, se a única preocupação da organização fosse com as normas requeridas na otimização do motor, os engenheiros da primeira concepção seriam preferíveis aos das duas outras. Além disso, a empresa poderia rever a função dos engenheiros da terceira concepção. Tais engenheiros são capazes de se colocar no lugar de outros (clientes), portanto estão, de certa forma, mais preparados para assumir uma função gerencial. A organização deve, portanto, fazer a seguinte pergunta: em qual função a pessoa que está num determinado estágio de desenvolvimento maximiza o valor da empresa? Infere-se, dessa forma, que a perspectiva desenvolvimentista permite um melhor ajuste entre o desenvolvimento das competências individuais, ou seja, do próprio indivíduo, e os objetivos organizacionais.

Finalmente, ao considerarem a capacidade de construir significados como o ponto central do desenvolvimento, as teorias do desenvolvimento abrangem todas as dimensões do indivíduo. Isso vai ao encontro das novas demandas sociais impostas pela sociedade do conhecimento (TUOMI, 2001).

\begin{abstract}
The interaction pattern between organizations within digital era is non-linear. This generates an environment that requires continuous knowledge creation by organizations. Such requirement demands behaviour and performance changes by individuals, having repercussions in organizational knowledge creation. From this perspective, it is proposed that developmental theories be applied to organizational knowledge creation. Thus, organizations can both optimize knowledge creation and take into account individual development. Main developmental theories are introduced. Their applicability is demonstrated on leadership development. Once having theoretical foundation, the four knowledge conversion processes are analysed in the framework of developmental theories. One of the conclusions in this work is that the capacity of creating explict knowledge, as considering the individual, is limited by a tacit counterbalance. It is also inferred that developmental framework makes possible a better fit between individual competence development and organizational goals.
\end{abstract}

Keywords: knowledge creation; developmental theories; leadership; non-linear systems. 


\section{Referências}

COOK-GREUTER, S. R. Making the case for a developmental perspective. Industrial and Commercial Training, v. 36, n. 7, p. $275-281,2004$.

COOK-GREUTER, S. R. Postautonomous ego development: a study of its nature and measurement. 1999. Thesis (Doctor of Philosofy in Education) - Graduate School of Education of Harvard University, Boston, 1999.

ERIKSON, E. O Ciclo de Vida Completo. Porte Alegre: Artes Médicas, 1998.

EVANS, P. B.; WURSTER, Thomas S. Strategy and the new economics of information. Harvard Business Review, v. 75, n. 5, p. 71-82, 1997.

FISHER, D.; ROOKE, D.; TORBERT, B. Personal and Organizational Transformations. 4. ed. Edge $\backslash$ Work Press, 2003 .

ISHIKAWA, G. A relação entre os estágios do desenvolvimento de Loevinger e o desenvolvimento da liderança. Dissertação (mestrado) - Engenharia de Produção, Universidade Federal de Santa Catarina, Florianópolis, 2005.

KEGAN, R. The evolving self: problem and process in human development. Cambridge: Harvard University Press, 1982.

LOEVINGER, J. Measurement of personality: true or false. Psychological Inquiry, v. 4, n. 1, p. 1-16, 1993.

NONAKA, I.; TAKEUCHI, H. Criação de Conhecimento na Empresa. Rio de Janeiro: Campus, 1997.

STACEY, R. D. Complexity and Creativity in Organizations. San Francisco: Berret-Koehler Publishers, 1996.

STACEY, R.D. The science of complexity: an alternative perspective for strategic change processes. Strategic Management Journal, v. 16, n. 6, p. 477-495, 1995.

ROOKE, D. Organisational transformation requires the presence of leaders who are strategists and magicians. Organisations and People, v. 4, n. 3, 1997.

ROOKE, D.; TORBERT, W. R. Organizational transformation as function of CEOs' developmental stage. Organization Development Journal, v. 16, n. 1, p. 11-28, 1998.

ROOKE, D.; TORBERT, W. R. Sete transformações da liderança. Harvard Business Review, vol. 83, n. 4, p. 45-54, 2005.

SANDBERG, J. Understanding human competence at work: an interpretative approach. Academy of Management Journal, v. 43, n. 1, p. 9-25, 2000.

TUOMI, I. From periphery to center: emerging research topics on knowledge society. Helsinki: Tekes, 2001.

\section{Dados completos de todos os autores:}

\section{Nome completo: Carlos Henrique Prim}

Filiação institucional: Universidade Federal de Santa Catarina

Departamento: Engenharia e Gestão do Conhecimento

Função ou cargo ocupado: Doutorando

Endereço completo para correspondência: Servidão José Ventura, 35, Bairro Trindade, CEP 88036642, Florianópolis/SC, Brasil 
Telefones para contato: (48) 3234-3173 / 9951-7213

e-mail: chprim@hotmail.com

\section{Nome completo: Cristiano José Castro de Almeida Cunha}

Filiação institucional: Universidade Federal de Santa Catarina

Departamento: Engenharia de Produção

Função ou cargo ocupado: Professor

Endereço completo para correspondência: Rua Capitão Romualdo de Barros, 795, Casa 15, Bairro Carvoeira, 88040-600, Florianópolis/SC, Brasil

Telefones para contato: (48) 3334-4601 / 9972-4197

e-mail:01cunha@gmail.com

Recebido para publicação em: 10/07/06

Aceito para publicação em: 11/09/06 